\title{
La vivienda sostenible, desde un enfoque teórico y de política pública en Colombia*
}

\author{
Diana Elizabeth Valencia**
}

\author{
Recibido: 07/09/2017 • Aceptado: 02/04/2018 \\ https://doi.org/10.22395/rium.v17n33a2
}

\begin{abstract}
Resumen
La vivienda social en Colombia no incluye, como parámetros de diseño, criterios de orden ambiental, ni social pues prima el cierre financiero, es por esto que el objetivo del artículo es proponer lineamientos para el diseño de prototipos de vivienda de interés social (VIS) sostenible, a partir de la aplicación de la matriz línea base.

Respecto a la metodología empleada, para el ejercicio de esta investigación se parte de la matriz elaborada en el proyecto de investigación denominado Propuesta de lineamientos para el diseño arquitectónico y construcción de un prototipo VIS para Medellín, que incluya en su diseño criterios de sostenibilidad a partir de la línea base elaborada en la fase II y aplicable a Medellín y el Área Metropolitana. Esta matriz está compuesta por cuatro ejes temáticos: selección del sitio o emplazamiento, uso racional del agua, materiales y habitabilidad. Luego de aplicada, los resultados sirvieron para definir lineamientos de intervención en las diferentes escalas.

El proyecto arrojó como resultados unos lineamientos de intervención que pueden ser aplicados en la etapa de diseño de este tipo de proyectos o que pueden ser utilizados como punto de referencia para evaluar la sostenibilidad en los proyectos de VIS desarrollados.
\end{abstract}

Palabras clave: habitabilidad; sostenibilidad; desarrollo humano; vivienda social.

Artículo de investigación desarrollado en el marco del proceso de investigación de la formación doctoral de la autora, en Criterios Territoriales y Urbanos, llevado a cabo en el Instituto Superior Politécnico José Antonio Echeverría (ISPJAE), La Habana, Cuba, dirigida por el Dr. Ing. Arq. Rubén Andrés Bancrofft Hernández y elaborada entre octubre del año 2013 y junio de 2017.

** M. Sc. Profesora de tiempo completo de la Universidad de San Buenaventura, Medellín, Colombia. Carrera 56C N. 51-110. Teléfono: +57+4 51475600. Correo electrónico: diana.valencia@usbmed.edu.co. Orcid: https://orcid. org/0000-0002-4542-4128 


\title{
Sustainable Housing, from a Theoretical and Public Policy Approach in Colombia
}

\begin{abstract}
Social housing in Colombia does not include, as design parameters, environmental or social criteria, since financial closure is a priority. This is why the objective of this article is to propose guidelines for the design of prototypes of sustainable social housing (VIS), based on the application of the baseline matrix.

With respect to the methodology used, this research is based on the matrix elaborated in the research project called Proposal for Guidelines for the Architectural Design and Construction of a VIS Prototype for Medellin, which includes sustainability criteria in its design based on the baseline elaborated in phase II and applicable to Medellin and the Metropolitan Area. This matrix is composed of four thematic axes: site selection, rational use of water, materials and habitability. Once applied, the results served to define guidelines for intervention at the different scales.

The project resulted in intervention guidelines that can be applied in the design stage of this type of project or that can be used as a reference point to evaluate sustainability in VIS projects developed.
\end{abstract}

Keywords: habitability; sustainability; human development; social housing.

\section{A moradia sustentável a partir de um enfoque teórico e de política pública na Colômbia}

\section{Resumo}

A moradia social na Colômbia não inclui, como parâmetro de desenho, critérios de ordem ambiental nem social, pois prima pelo fechamento financeiro. Por esse motivo, o objetivo do artigo é propor alinhamentos para o desenho de protótipos de habitação de interesse social (HIS) sustentável a partir da aplicação da matriz linha base.

Com respeito à metodologia empregada, para o exercício dessa pesquisa parte-se da matriz elaborada no projeto de pesquisa denominado Proposta de alinhamentos para o desenho arquitetônico e construção de um protótipo HIS para Medellín que inclua em seu desenho critérios de sustentabilidade a partir da linha base elaborada na fase II e aplicável a Medellín e Área Metropolitana. Essa matriz é composta por quatro eixos temáticos: seleção do lugar ou posicionamento, uso racional da água, materiais e habitabilidade. Após a aplicação, os resultados serviram para definir alinhamentos de intervenção nas diferentes escalas.

O projeto teve como resultados alguns alinhamentos de intervenção que podem ser aplicados na etapa de desenho desse tipo de projetos ou que podem ser utilizados como ponto de referência para avaliar a sustentabilidade nos projetos de HIS desenvolvimentos.

Palavras-chave: habitabilidade; sustentabilidade; desenvolvimento humano; moradia social. 


\section{INTRODUCCIÓN}

En este artículo se recogen los resultados iniciales del proceso de investigación realizado como parte de la formación doctoral de la autora, cuya temática son los criterios territoriales y urbanos aplicados en la etapa de diseño de un proyecto, para la evaluación de la sostenibilidad ambiental de la vivienda social desarrollada en Colombia bajo la tipología de multifamiliares (caso de estudio Medellín), y da cuenta de los resultados alcanzados en la primera etapa de la tesis doctoral.

En general, en los países latinoamericanos, la mayor problemática de orden habitacional se concentra en la vivienda social o vivienda para población de bajos ingresos. Sin embargo, la inversión que se destina a dicha infraestructura no es proporcional a la magnitud del problema. Es por esto que las soluciones arquitectónicas entregadas por el Estado carecen de las siguientes condiciones, que son vitales para dar cuenta de un hábitat integral y sostenible: relación con el contexto cultural, social y biofísico, concepción de la vivienda como un espacio para el habitar que implica una cualificación del mismo y una relación con el entorno y como el ámbito que posibilita el desarrollo humano.

Las soluciones arquitectónicas a través de las cuales se resuelve el déficit habitacional de las poblaciones de bajos recursos distan mucho de los factores antes mencionados y canalizan su respuesta en una ecuación simple en la que el dinero se convierte en metros cuadrados. Pero esta misma ecuación no es garantía, ni para la población ni para el Estado, de ser la respuesta integral a un problema de múltiples aristas y prioritario en cuanto a la calidad de vida.

Es por esto que a través de este artículo se presentan aquellos criterios o elementos que, derivados del análisis teórico y de política pública, siguen vigentes en la propuesta de sostenibilidad de la vivienda social en Colombia, desde una mirada integral del hábitat.

\section{MATERIALES Y MÉTODOS}

El proceso investigativo del cual se deriva el presente artículo (primera etapa), se concentra en un análisis documental que elabora un marco teórico y normativo de carácter internacional y nacional aplicable a la vivienda social en Colombia, de tal manera que arroje luces sobre los aspectos relevantes en el análisis de dicha tipología arquitectónica. Se realiza un estudio cualitativo de los diferentes conceptos buscando correlacionar los mismos, de tal manera que el proceso analítico arroje elementos en común a considerar en un modelo teórico que evalúe la sostenibilidad ambiental en la vivienda.

Se iniciará con la conceptualización de los términos vivienda y sostenibilidad, y su interpretación en el contexto internacional y nacional para, posteriormente, ponerla 
en contraste con lo especificado en el marco normativo que regula las actuaciones que en materia de vivienda se desarrollan en el país.

\section{REFERENTES CONCEPTUALES DE ORDEN INTERNACIONAL}

Cuando se pretende entender el término de sostenibilidad se hace necesario remontar el concepto propuesto en la Cumbre de la Tierra de 1992, Conferencia de la Organización de las Naciones Unidas que, partiendo de una visión economicista, define el desarrollo sostenible como aquel que garantiza el crecimiento económico y el bienestar social sin poner en riesgo la conservación y preservación de los recursos naturales para las generaciones futuras [1].

El informe Brundtland planteó que "el desarrollo sostenible implica límites, no límites absolutos, sino limitaciones que el estado actual de la tecnología y de la organización social, así como la capacidad de la biosfera de absorber los efectos de las actividades humanas, imponen a los recursos del medio ambiente" [2].

Una de las grandes conclusiones arrojadas por la Cumbre de la Tierra y por el Informe Brundtland, fue la necesidad de generar cambios en los modelos de desarrollo y comprometer a los diferentes sectores productivos a incorporar procesos de gestión ambiental en el desarrollo de su actividad. Una de las actividades productivas que mayor impacto genera sobre el ambiente es la actividad constructora, es por esto que el gremio de arquitectos en el marco del congreso celebrado por la Unión Internacional de Arquitectos en 1993, estableció la corresponsabilidad en la crisis ambiental por los altos impactos de la industria de la construcción. Adicionalmente, se incorporó un nuevo concepto como directriz de actuación derivado del compromiso del congreso así: "el diseño sostenible integra consideraciones de eficiencia en el uso de recursos y de la energía, ha de producir edificios sanos, ha de utilizar materiales ecológicos y debe considerar la sensibilidad estética que inspire, afirme y emocione" [3].

"En conjunto con el Programa de Naciones Unidas para el Ambiente -PNUMAdesarrolló en 1992 la Agenda 21 para la Construcción Sostenible en Países en Desarrollo, en la cual, se definió el concepto de construcción sostenible de la siguiente manera: la construcción sostenible significa que los principios del desarrollo sostenible son aplicados al proceso de la construcción del entorno construido (durante todo su ciclo de vida), desde la extracción de los materiales, pasando por la planeación, diseño y construcción de los edificios y la infraestructura, hasta su demolición final y la gestión de sus residuos. Es un proceso holístico que apunta a restaurar y mantener la armonía entre los sistemas naturales y el entorno construido, mientras se construyen ciudades que reafirmen la dignidad humana e impulsen la equidad económica" [4].

En la tabla 1, se presenta la síntesis de los conceptos que se asocian a los términos de arquitectura y sostenibilidad. 
Tabla 1. Conceptos asociados a la arquitectura sostenible

\begin{tabular}{|c|c|c|c|}
\hline Denominación & Objetivo & $\begin{array}{c}\text { Criterios o variables que } \\
\text { considera }\end{array}$ & Observaciones \\
\hline $\begin{array}{l}\text { Arquitectura ecológi- } \\
\text { ca [10]. Concepto to- } \\
\text { mado del documento: } \\
\text { Criterios } \\
\text { ambientales para el } \\
\text { diseño y construcción } \\
\text { de vivienda urbana. }\end{array}$ & $\begin{array}{l}\text { Propende por la cuida- } \\
\text { dosa inserción de las } \\
\text { construcciones en el en- } \\
\text { torno natural, buscando } \\
\text { que su emplazamiento } \\
\text { genere el menor impac- } \\
\text { to nocivo posible per- } \\
\text { mitiendo la coexistencia } \\
\text { armónica entre el lugar, } \\
\text { el edificio y el hombre } \\
\text { que lo habita. }\end{array}$ & $\begin{array}{l}\text { Esta arquitectura tiene } 10 \\
\text { principios básicos: } \\
\text { Valorar el sitio y las necesida- } \\
\text { des constructivas. } \\
\text { Proyectar la obra de acuerdo } \\
\text { con el clima local. } \\
\text { Ahorrar energía. } \\
\text { Pensar en fuentes de energía } \\
\text { renovables. } \\
\text { Ahorrar agua. } \\
\text { Construir edificios de mayor } \\
\text { calidad. } \\
\text { Evitar riesgos para la salud. } \\
\text { Utilizar materiales obtenidos } \\
\text { de materias primas generadas } \\
\text { localmente. } \\
\text { Utilizar materiales reciclables. } \\
\text { Gestionar ecológicamente los } \\
\text { desechos. }\end{array}$ & $\begin{array}{l}\text { Reflexiona sobre el im- } \\
\text { pacto ambiental de todos } \\
\text { los procesos implicados } \\
\text { en la construcción y pues- } \\
\text { ta en operación de la mis- } \\
\text { ma, pasando por la ex- } \\
\text { tracción de materiales, el } \\
\text { proceso constructivo y la } \\
\text { implantación en el lugar. }\end{array}$ \\
\hline $\begin{array}{l}\text { Arquitectura biocli- } \\
\text { mática. [10] } \\
\text { Concepto tomado del } \\
\text { documento: Criterios } \\
\text { ambientales para el } \\
\text { diseño y construcción } \\
\text { de vivienda urbana. }\end{array}$ & $\begin{array}{l}\text { Generar espacios con } \\
\text { óptimas condiciones de } \\
\text { confort y bienestar, in- } \\
\text { corporando determi- } \\
\text { nantes de diseño que } \\
\text { permitan la interrela- } \\
\text { ción de variables climá- } \\
\text { ticas para lograrlo. }\end{array}$ & $\begin{array}{l}\text { Clima. } \\
\text { Condiciones del entorno. } \\
\text { Confort térmico en el interior } \\
\text { de la vivienda. }\end{array}$ & $\begin{array}{l}\text { Se concentra exclusiva- } \\
\text { mente en el diseño y los } \\
\text { elementos arquitectóni- } \\
\text { cos, sin necesidad de uti- } \\
\text { lizar sistemas mecánicos } \\
\text { complejos. }\end{array}$ \\
\hline $\begin{array}{l}\text { Construcción sosteni- } \\
\text { ble. [10] } \\
\text { Concepto tomado del } \\
\text { documento: Criterios } \\
\text { ambientales para el } \\
\text { diseño y construcción } \\
\text { de vivienda urbana. }\end{array}$ & $\begin{array}{l}\text { Una construcción sos- } \\
\text { tenible es aquella que } \\
\text { está en sincronía con el } \\
\text { sitio, hace uso de ener- } \\
\text { gía, agua y materiales } \\
\text { de un modo eficiente y } \\
\text { provee confort y salud a } \\
\text { sus usuarios. Todo esto } \\
\text { es alcanzado gracias a } \\
\text { un proceso de diseño } \\
\text { consciente del clima y } \\
\text { la ecología del entorno } \\
\text { donde se construye la } \\
\text { edificación [5]. }\end{array}$ & $\begin{array}{l}\text { Eficiencia energética. } \\
\text { Eficiencia en agua. } \\
\text { Materiales de construcción } \\
\text { de baja energía embebida. } \\
\text { Calidad del ambiente interior. } \\
\text { Sostenibilidad del emplaza- } \\
\text { miento. } \\
\text { Edificaciones y entorno ex- } \\
\text { terior. } \\
\text { Sostenibilidad urbana. }\end{array}$ & $\begin{array}{l}\text { A pesar de que la guía co- } \\
\text { lombiana menciona múl- } \\
\text { tiples aspectos a consi- } \\
\text { derar en una construc- } \\
\text { ción sostenible, se deci- } \\
\text { dió que los siguientes as- } \\
\text { pectos fueran tomados en } \\
\text { la primera fase de crea- } \\
\text { ción de la guía nacional de } \\
\text { construcción sostenible: } \\
\text { Eficiencia energética de } \\
\text { las edificaciones. } \\
\text { Eficiencia del uso de agua } \\
\text { en las edificaciones [5]. }\end{array}$ \\
\hline
\end{tabular}




\begin{tabular}{|c|c|c|c|}
\hline Denominación & Objetivo & $\begin{array}{c}\text { Criterios o variables que } \\
\text { considera }\end{array}$ & Observaciones \\
\hline $\begin{array}{l}\text { Arquitectura sosteni- } \\
\text { ble. [10] } \\
\text { Concepto tomado del } \\
\text { documento: Criterios } \\
\text { ambientales para el } \\
\text { diseño y construcción } \\
\text { de vivienda urbana. }\end{array}$ & $\begin{array}{l}\text { Aquella que tiene en } \\
\text { cuenta el impacto que } \\
\text { va a tener el edificio du- } \\
\text { rante todo su ciclo de } \\
\text { vida, desde su construc- } \\
\text { ción, pasando por su uso } \\
\text { y su derribo final. }\end{array}$ & $\begin{array}{l}\text { 1. Ubicación adecuada. } \\
\text { 2. Integración en su entorno } \\
\text { más próximo. } \\
\text { 3. Aplicación de variables } \\
\text { bioclimáticas. } \\
\text { 4. Uso de materiales de cons- } \\
\text { trucción, que involucren } \\
\text { aspectos de disponibilidad, } \\
\text { estética y accesibilidad. } \\
\text { 5. Utilización de materiales } \\
\text { y tecnologías que tengan } \\
\text { la menor cantidad de } \mathrm{CO}_{2} \\
\text { en el entero ciclo de vida. } \\
\text { 6. Implementación de siste- } \\
\text { mas energéticos alternati- } \\
\text { vos. } \\
\text { 7. Implantar circuitos cerra- } \\
\text { dos de aguas y residuos. }\end{array}$ & $\begin{array}{l}\text { Presenta una mirada más } \\
\text { abarcante de la sostenibi- } \\
\text { lidad, incluyendo el con- } \\
\text { texto físico biótico como } \\
\text { un elemento determinan- } \\
\text { te y de integración con el } \\
\text { entorno. }\end{array}$ \\
\hline $\begin{array}{l}\text { Conferencia de las } \\
\text { Naciones Unidas Há- } \\
\text { bitat I [6], tomado de: } \\
\text { Satisfacción residen- } \\
\text { cial en la vivienda bá- } \\
\text { sica, Serviu: la pers- } \\
\text { pectiva de capital so- } \\
\text { cial. }\end{array}$ & $\begin{array}{l}\text { La vivienda es entendi- } \\
\text { da no solamente como } \\
\text { una unidad que cobija a } \\
\text { una familia, sino como } \\
\text { un sistema integral, que } \\
\text { comprende también el } \\
\text { terreno, la infraestruc- } \\
\text { tura para los servicios de } \\
\text { urbanización y servicios } \\
\text { y el equipamiento social } \\
\text { y comunitario, dentro de } \\
\text { un contexto cultural, so- } \\
\text { cio-económico, político } \\
\text { y físico ambiental. }\end{array}$ & $\begin{array}{l}\text { La vivienda se manifestó en } \\
\text { diversas escalas y lugares, que } \\
\text { son: localización urbanas o } \\
\text { rurales, vecindarios, conjun- } \\
\text { tos habitacionales, entornos y } \\
\text { unidades de vivienda. }\end{array}$ & $\begin{array}{l}\text { Los diversos atributos } \\
\text { son expresados en aspec- } \\
\text { tos funcionales, espacia- } \\
\text { les, formales (estéticos y } \\
\text { significado), materiales y } \\
\text { ambientales [6]. }\end{array}$ \\
\hline $\begin{array}{l}\text { Concepto de hábitat } \\
\text { en el modelo chile- } \\
\text { no [6] tomado de: } S a \text { - } \\
\text { tisfacción residencial } \\
\text { en la vivienda básica, } \\
\text { Serviu: la perspectiva } \\
\text { de capital social. }\end{array}$ & $\begin{array}{l}\text { "Puede entenderse } \\
\text { como la percepción y } \\
\text { valoración que diversos } \\
\text { observadores y partici- } \\
\text { pantes le asignan al to- } \\
\text { tal y a los componentes } \\
\text { de un conjunto habita- } \\
\text { cional, en cuanto a sus } \\
\text { diversas propiedades o } \\
\text { atributos en sus interac- } \\
\text { ciones mutuas y con el } \\
\text { contexto en el cual se } \\
\text { inserta". }\end{array}$ & $\begin{array}{l}\text { "El concepto de hábitat esta- } \\
\text { blece distintas jerarquizacio- } \\
\text { nes de acuerdo con variables } \\
\text { de orden axiológico, sicoso- } \\
\text { cial, cultural, económico y } \\
\text { político" [6]. }\end{array}$ & $\begin{array}{l}\text { El concepto de hábitat } \\
\text { propuesto por el mo- } \\
\text { delo chileno enfatiza } \\
\text { en el componente hu- } \\
\text { mano, anteponiendo el } \\
\text { mismo a la respuesta de } \\
\text { infraestructura. }\end{array}$ \\
\hline
\end{tabular}




\begin{tabular}{|c|c|c|c|}
\hline Denominación & Objetivo & $\begin{array}{c}\text { Criterios o variables que } \\
\text { considera }\end{array}$ & Observaciones \\
\hline $\begin{array}{l}\text { Desarrollo sistémico } \\
\text { de la política de vi- } \\
\text { vienda en Cuba [7], } \\
\text { tomado del texto De- } \\
\text { sarrollo sistémico de } \\
\text { la política de vivien- } \\
\text { da en Cuba. }\end{array}$ & $\begin{array}{l}\text { "La vivienda no puede } \\
\text { considerarse como un } \\
\text { hecho aislado, deberá } \\
\text { enfocarse como un sis- } \\
\text { tema que garantice una } \\
\text { visión coherente, inte- } \\
\text { gral, con la necesaria } \\
\text { interrelación entre las } \\
\text { políticas y estrategias } \\
\text { sociales, de vivienda, } \\
\text { de planeamiento, urba- } \\
\text { nística, constructiva y } \\
\text { de producción de mate- } \\
\text { riales, entre otras, bajo } \\
\text { un principio general de } \\
\text { desarrollo sustentable. } \\
\text { Implicados [7]. }\end{array}$ & $\begin{array}{l}\text { Espacios suficientes que satis- } \\
\text { fagan los estilos de vida y la } \\
\text { diversidad familiar. } \\
\text { Servicios básicos (agua, al- } \\
\text { cantarillado, entre otros), } \\
\text { servicios extradomiciliarios } \\
\text { (alimentación, educación, } \\
\text { salud), } \\
\text { calidad ambiental, } \\
\text { condiciones de habitabilidad } \\
\text { acordes con el desarrollo } \\
\text { social en educación, salud, } \\
\text { cultura, deporte. }\end{array}$ & $\begin{array}{l}\text { Ese enfoque sistémico, } \\
\text { multidimensional de- } \\
\text { berá combinar de ma- } \\
\text { nera equilibrada las } \\
\text { tres dimensiones: eco- } \\
\text { nómica, social y am- } \\
\text { biental. Además, será } \\
\text { un proceso multidisci- } \\
\text { plinario y con multipli- } \\
\text { cidad de actores. }\end{array}$ \\
\hline
\end{tabular}

Fuente: elaboración propia

Observando los objetivos y criterios planteados por los cuatro conceptos descritos en la tabla 1, se encuentra que coinciden en la necesidad de garantizar una selección de materiales de construcción que generen un mínimo impacto ambiental y la necesidad de presentar una propuesta para el manejo de los residuos de la construcción. En el caso de la arquitectura bioclimática, esta concentra su actuar en el confort térmico. Podría decirse que el concepto que mira, de manera más amplia, la sostenibilidad, es el de arquitectura sostenible, sin embargo, hace énfasis de manera especial en resolver los requerimientos funcionales de la arquitectura y la demanda que deriva de esta en términos de consumo de recursos, pero deja de lado aspectos de orden sociocultural y económico claves según se plantea en la siguiente cita: "el desarrollo verdaderamente sostenible pasa por una sociedad más equitativa, donde hayan disminuido significativamente las desigualdades de género y en la cual los objetivos y metas a lograr estén bien definidas y se correspondan para todos. La sostenibilidad en su dimensión social permanece todavía pobremente definida, y sus vínculos o articulación con el componente ecológico o ambiental pendientes de ser esclarecidos" [8].

A continuación, en la tabla 2 se presenta el análisis sistémico de los referentes conceptuales mencionados anteriormente. 
Tabla 2. Análisis sistémico de los referentes conceptuales.

\begin{tabular}{|l|l|l|l|}
\hline \multicolumn{1}{|c|}{ Sistema } & \multicolumn{1}{|c|}{ Concepto } & \multicolumn{1}{|c|}{ Temáticas relacionadas } & \multicolumn{1}{c|}{ Observaciones } \\
\hline Múltiples sistemas & $\begin{array}{l}\text { Arquitectura } \\
\text { sostenible [10] }\end{array}$ & $\begin{array}{l}\text { Lectura del contexto: biofísi- } \\
\text { co y físico biótico. }\end{array}$ & $\begin{array}{l}\text { Presenta una mirada integral } \\
\text { de los determinantes físicos. }\end{array}$ \\
\cline { 2 - 4 } & $\begin{array}{l}\text { Hábitat I } \\
(1976)[6]\end{array}$ & $\begin{array}{l}\text { Contexto cultural, socio-eco- } \\
\text { nómico, político y fisicoam- } \\
\text { biental. }\end{array}$ & $\begin{array}{l}\text { Los diversos atributos son } \\
\text { expresados en aspectos fun- } \\
\text { cionales, espaciales, forma- } \\
\text { les (estéticos y significado), } \\
\text { materiales y ambientales [6]. }\end{array}$ \\
\cline { 2 - 5 } & $\begin{array}{l}\text { Construcción } \\
\text { sostenible [5] }\end{array}$ & $\begin{array}{l}\text { Sincronía con el sitio, uso efi- } \\
\text { ciente de energía, agua y ma- } \\
\text { teriales, confort, } \\
\text { salud. }\end{array}$ & $\begin{array}{l}\text { Articula las dimensiones fi- } \\
\text { sicoespacial, biofísica y so- } \\
\text { ciocultural. }\end{array}$ \\
\hline \multirow{2}{*}{ Biofísico o ecológico } & $\begin{array}{l}\text { Arquitectura } \\
\text { ecológica [10] }\end{array}$ & $\begin{array}{l}\text { Consumo de recursos natu- } \\
\text { rales. } \\
\text { Producción de desechos. }\end{array}$ & $\begin{array}{l}\text { Evalúa lo impactos generados } \\
\text { por el proceso constructivo. }\end{array}$ \\
\cline { 2 - 5 } & $\begin{array}{l}\text { Arquitectu- } \\
\text { ra bioclimáti- } \\
\text { ca [10] }\end{array}$ & $\begin{array}{l}\text { Clima como determinante } \\
\text { para el diseño. }\end{array}$ & $\begin{array}{l}\text { Prioriza el confort como cri- } \\
\text { terio director. }\end{array}$ \\
\hline
\end{tabular}

Fuente: elaboración propia

El tomar la decisión de realizar un abordaje para el análisis del problema desde un enfoque sistémico, parte de reconocer cómo cada una de las dimensiones está respaldada en referentes de orden nacional e internacional que la han destacado como de relevancia, tanto para la comprensión del problema como para la generación de estrategias de intervención. En la tabla 3, se presentan las dimensiones y factores.

Tabla 3. Factores incluidos en el análisis sistémico por dimensiones

\begin{tabular}{|c|c|c|}
\hline Dimensión & Referente & Factores incluidos \\
\hline $\begin{array}{l}\text { Mirada multi- } \\
\text { dimensional }\end{array}$ & Usaid, 2013 [4]. & $\begin{array}{l}\text { "Es un proceso holístico que apunta a restaurar y mantener } \\
\text { la armonía entre los sistemas naturales y el entorno cons- } \\
\text { truido, mientras se construyen ciudades que reafirmen la } \\
\text { dignidad humana e impulsen la equidad económica" [4]. }\end{array}$ \\
\hline & $\begin{array}{l}\text { Metodología ciudades emer- } \\
\text { gentes y sostenibles (CES) } \\
\text { (Banco Interamericano de De- } \\
\text { sarrollo), } 2016 . \\
\text { Pretende abordar el análisis de } \\
\text { la sostenibilidad de las ciuda- } \\
\text { des en tres dimensiones }\end{array}$ & $\begin{array}{l}\text { Dimensión del riesgo de desastres y cambio climático: } \\
\text { Gerencia ambiental } \\
\text { Control de la contaminación local } \\
\text { Mitigación del cambio climático y la prevención y re- } \\
\text { ducción de la vulnerabilidad a los desastres naturales. } \\
\text { Dimensión del desarrollo urbano integral: }\end{array}$ \\
\hline
\end{tabular}




\begin{tabular}{|c|c|c|}
\hline Dimensión & Referente & Factores incluidos \\
\hline & $\begin{array}{l}\text { fundamentales: i) el riesgo de } \\
\text { desastres y cambio climático, } \\
\text { ii) el desarrollo urbano inte- } \\
\text { gral y iii) el ámbito fiscal y las } \\
\text { cuestiones de gobernabilidad y } \\
\text { transparencia [9]. }\end{array}$ & $\begin{array}{l}\text { diseño de la ciudad y a su huella, (desigualdad social y } \\
\text { la distribución desequilibrada de los servicios, la efica- } \\
\text { cia del transporte urbano, la competitividad y la seguri- } \\
\text { dad ciudadana). } \\
\text { Dimensión fiscal: y capacidad del gobierno local de fi- } \\
\text { nanciar las inversiones necesarias, lograr la prestación } \\
\text { de los servicios, controlar correctamente sus gastos y su } \\
\text { deuda y tomar decisiones de una manera transparente, } \\
\text { planificada y eficaz. }\end{array}$ \\
\hline \multirow[t]{4}{*}{$\begin{array}{l}\text { Biofisica o } \\
\text { ecológica }\end{array}$} & $\begin{array}{l}\text { Criterios ambientales para el di- } \\
\text { seño y construcción de vivien- } \\
\text { da urbana, } 2012[5] .\end{array}$ & $\begin{array}{l}\text { Criterios ambientales para la producción y uso de la vi- } \\
\text { vienda [10]: } \\
\text { Agua. } \\
\text { Suelo. } \\
\text { Materiales. } \\
\text { Energía. }\end{array}$ \\
\hline & $\begin{array}{l}\text { Comisión Nacional de Medio } \\
\text { Ambiente del gobierno de Chi- } \\
\text { le, } 2007 \text { [11]. }\end{array}$ & $\begin{array}{l}\text { Balance entre la oferta ambiental y el consumo de re- } \\
\text { cursos. }\end{array}$ \\
\hline & $\begin{array}{l}\text { Documento ejecutivo políti- } \\
\text { ca pública de ecourbanismo y } \\
\text { construcción sostenible de Bo- } \\
\text { gotá, } 2014 \text { [12]. }\end{array}$ & $\begin{array}{l}\text { Desde una perspectiva ecosistémica se consideran cua- } \\
\text { tro ciclos urbanos básicos: el hidrológico, atmosférico, } \\
\text { energético y de la materia orgánica y los residuos [13]. }\end{array}$ \\
\hline & $\begin{array}{l}\text { Metodología ciudades emer- } \\
\text { gentes y sostenibles (CES) } \\
\text { (Banco Interamericano de De- } \\
\text { sarrollo), } 2016 \text { [9]. }\end{array}$ & $\begin{array}{l}\text { Dimensión del riesgo de desastres y cambio climático: } \\
\text { Gerencia ambiental. } \\
\text { Control de la contaminación local. } \\
\text { Mitigación del cambio climático y la prevención y re- } \\
\text { ducción de la vulnerabilidad a los desastres naturales. }\end{array}$ \\
\hline \multirow[t]{4}{*}{ Fisicoespacial } & $\begin{array}{l}\text { Modelo de indicadores de ur- } \\
\text { banismo ecológico, Agencia de } \\
\text { Ecología Urbana de Barcelona, } \\
2013 \text { [14]. }\end{array}$ & $\begin{array}{l}\text { Plantea cuatro objetivos básicos de la sostenibilidad ur- } \\
\text { bana: 1) cohesión social, 2) eficiencia, 3) complejidad y } \\
\text { 4) compacidad y funcionalidad. }\end{array}$ \\
\hline & $\begin{array}{l}\text { Decreto } 2190 \text { de } 2008 \text {, en su ar- } \\
\text { tículo } 2[15] .\end{array}$ & $\begin{array}{l}\text { Solucion de vivienda: habitación en condiciones sanita- } \\
\text { rias satisfactorias de espacio, servicios públicos y cali- } \\
\text { dad de la estructura. }\end{array}$ \\
\hline & $\begin{array}{l}\text { Documento técnico de soporte } \\
\text {-recomendaciones de política } \\
\text { pública de construcción y ur- } \\
\text { banismo sostenible- informe } \\
\text { final, 2013, p. } 197 \text { [4]. }\end{array}$ & $\begin{array}{l}\text { Cuatro componentes del entorno construido: o infraes- } \\
\text { tructura vial y de servicios públicos o espacio público o } \\
\text { VIS (construcción) o equipamientos públicos. }\end{array}$ \\
\hline & $\begin{array}{l}\text { Decreto } 1285 \text { de } 2015 \text {. Li- } \\
\text { neamientos de construcción } \\
\text { sostenible para edificaciones } \\
{[16] .}\end{array}$ & $\begin{array}{l}\text { Porcentajes obligatorios de ahorro en agua y energía } \\
\text { según clima y tipo de edificaciones. } \\
\text { Sistema de aplicación gradual para el territorio de con- } \\
\text { formidad con el número de habitantes de los municipios. }\end{array}$ \\
\hline
\end{tabular}




\begin{tabular}{|c|c|c|}
\hline Dimensión & Referente & Factores incluidos \\
\hline \multirow[t]{3}{*}{ Fisicoespacial } & & $\begin{array}{l}\text { Procedimiento para la certificación de la aplicación de } \\
\text { las medidas. } \\
\text { Procedimiento y herramientas de seguimiento y control a } \\
\text { la implementación de las medidas. Promoción de incenti- } \\
\text { vos a nivel local para la construcción sostenible. }\end{array}$ \\
\hline & $\begin{array}{l}\text { Metodología de evaluación in- } \\
\text { tegral sostenible urbana } \\
\text { (Portugal) [17] } \\
\text { Su objetivo principal es ser una } \\
\text { evaluación integral, equilibra- } \\
\text { da y justificada por los aspec- } \\
\text { tos importantes para generar } \\
\text { barrios sostenibles }\end{array}$ & $\begin{array}{l}\text { Morfología urbana: calidad del diseño de edificios, fiso- } \\
\text { nomía y calidad de imagen urbana, conectividad y mo- } \\
\text { vilidad, densidad de planificación. } \\
\text { Sintaxis urbana: edificios sostenibles, uso mixto de la } \\
\text { comunidad, infraestructura y servicios, estrategias de } \\
\text { futuro. }\end{array}$ \\
\hline & $\begin{array}{l}\text { Metodología ciudades emer- } \\
\text { gentes y sostenibles (CES) } \\
\text { (Banco Interamericano de De- } \\
\text { sarrollo), 2016, p. } 7 \text { [9]. }\end{array}$ & $\begin{array}{l}\text { Dimensión del riesgo de desastres y cambio climático: } \\
\text { Gerencia ambiental. } \\
\text { Control de la contaminación local. } \\
\text { Mitigación del cambio climático y la prevención y re- } \\
\text { ducción de la vulnerabilidad a los desastres naturales. } \\
\text { Dimensión del desarrollo urbano integral: } \\
\text { diseño de la ciudad y su huella, (desigualdad social y } \\
\text { la distribución desequilibrada de los servicios, la efica- } \\
\text { cia del transporte urbano, la competitividad y la seguri- } \\
\text { dad ciudadana. } \\
\text { Dimensión fiscal: y capacidad del gobierno local de fi- } \\
\text { nanciar las inversiones necesarias, lograr la prestación } \\
\text { de los servicios, controlar correctamente sus gastos y su } \\
\text { deuda y tomar decisiones de una manera transparente, } \\
\text { planificada y eficaz. }\end{array}$ \\
\hline \multirow[t]{2}{*}{$\begin{array}{l}\text { Sociocultural } \\
\text { (incluye lo le- } \\
\text { gal) }\end{array}$} & $\begin{array}{l}\text { Política pública de construcción } \\
\text { sostenible, Medellín, 2016 [18]. }\end{array}$ & $\begin{array}{l}\text { Viabilidad: relacion de costo-eficiencia economica de la } \\
\text { implementacion de lineamientos y estrategias de soste- } \\
\text { nibilidad en el ciclo de vida de los proyectos. }\end{array}$ \\
\hline & $\begin{array}{l}\text { Guía de asistencia técnica para } \\
\text { la vivienda de interés social N. }{ }^{\circ} \\
1,2011[19] .\end{array}$ & $\begin{array}{l}\text { El derecho a la vivienda adecuada, } \\
\text { seguridad jurídica de la tenencia, } \\
\text { disponibilidad de servicios, materiales e infraestructuras. } \\
\text { Gastos de vivienda soportables. } \\
\text { Vivienda habitable. } \\
\text { Vivienda asequible. } \\
\text { Lugar. } \\
\text { Adecuación cultural de la vivienda. }\end{array}$ \\
\hline Económica & $\begin{array}{l}\text { Lineamientos Ley de vivien- } \\
\text { da de } 2006 \text { de Mexico. Usaid, } \\
\text { p. } 140 \text { [4]. }\end{array}$ & $\begin{array}{l}\text { Promover seguridad jurídica y disminución de costos } \\
\text { de la vivienda. }\end{array}$ \\
\hline
\end{tabular}




\begin{tabular}{|c|c|c|}
\hline Dimensión & Referente & Factores incluidos \\
\hline & $\begin{array}{l}\text { Metodología ciudades emer- } \\
\text { gentes y sostenibles (CES) } \\
\text { (Banco Interamericano de De- } \\
\text { sarrollo), 2016. Pretende abor- } \\
\text { dar el análisis de la sostenibili- } \\
\text { dad de las ciudades en tres di- } \\
\text { mensiones } \\
\text { fundamentales: i) el riesgo de } \\
\text { desastres y cambio climático, } \\
\text { ii) el desarrollo urbano inte- } \\
\text { gral y iii) el ámbito fiscal y las } \\
\text { cuestiones de gobernabilidad y } \\
\text { transparencia, p. } 7 \text { [9]. }\end{array}$ & $\begin{array}{l}\text { Dimensión del desarrollo urbano integral: } \\
\text { diseño de la ciudad y su huella, (desigualdad social y } \\
\text { la distribución desequilibrada de los servicios, la efica- } \\
\text { cia del transporte urbano, la competitividad y la seguri- } \\
\text { dad ciudadana. } \\
\text { Dimensión fiscal: y capacidad del gobierno local de fi- } \\
\text { nanciar las inversiones necesarias, lograr la prestación } \\
\text { de los servicios, controlar correctamente sus gastos y su } \\
\text { deuda, y tomar decisiones de una manera transparente, } \\
\text { planificada y eficaz. }\end{array}$ \\
\hline
\end{tabular}

Fuente: elaboración propia

En la tabla 3 se observa cómo los diferentes estudios que abordan la problemática de la sostenibilidad en contextos urbanos y territoriales asumen el análisis desde cuatro dimensiones básicas: biofísica o ecológica, fisicoespacial, sociocultural y económica. El desarrollo conceptual llevado a cabo por Usaid como documento preparatorio de la política pública de urbanismo y construcción sostenible en Colombia, y la propuesta metodológica del BID de ciudades emergentes y sostenibles (CES), asumen una estrategia holística, que involucra una perspectiva sistémica e integral. Predominan las metodologías que se enfocan en la dimensión fisicoespacial, buscando resolver la relación entre el componente natural y construido a través del funcionamiento de la infraestructura física.

En el nivel local se han desarrollado varias guías desde el año 2011 encaminadas a dar lineamientos que apunten a condiciones de sostenibilidad en el ambiente construido, sin embargo, las mismas no pasan de hacer recomendaciones que pueden ser asumidas o no por los diferentes actores que participan del sector de la construcción.

A pesar de que el Decreto 1285 de 2015, denominado Lineamientos de construcción sostenible para edificaciones, muestra una mirada integral del problema partiendo de la definición de edificaciones sostenibles y considerando los siguientes aspectos: eficiencia energética, eficiencia en agua, materiales de construcción de baja energía embebida, calidad del ambiente interior, sostenibilidad del emplazamiento, edificaciones y entorno exterior y sostenibilidad urbana [16], solo se encuentra desarrollado en la Guía de construcción sostenible para el ahorro de agua y energía en edificaciones que se remite a una respuesta particular desde el medio físico que no genera articulaciones con otras dimensiones, ni propende por un enfoque holístico. De igual forma el decreto centra su atención en la sostenibilidad en edificaciones, y aunque incluye un apartado 
en relación con la sostenibilidad urbana, se observa en la guía su énfasis en el resultado final arquitectónico $[5,16]$.

\section{LAS POLITICAS PÚBLICAS COLOMBIANAS Y SU INCORPORACION DE LA SOSTENIBILI- DAD EN LA VIVIENDA}

La historia de la vivienda en Colombia, permite observar cómo han sido los criterios económicos los que han primado en la toma de decisiones, tanto en la selección del lugar como en el diseño arquitectónico de la vivienda, dejando ver que se ha entendido la vivienda como un hecho en el que prima lo arquitectónico y donde el análisis de la calidad de la misma desde el entorno y su relación con el contexto sociocultural no son aspectos importantes a integrar.

En la tabla 4, se presenta un ejercicio de comparación de las políticas públicas en Colombia en relación a la vivienda.

Tabla 4. Cuadro comparativo enfoque de las políticas públicas.

\begin{tabular}{|l|l|l|}
\hline \multicolumn{1}{|c|}{ Política pública } & \multicolumn{1}{|c|}{ Objetivo } & \multicolumn{1}{c|}{ Énfasis temático } \\
\hline $\begin{array}{l}\text { Constitución Política de } \\
\text { Colombia, 1991, artícu- } \\
\text { lo } 5 \text { [20]. }\end{array}$ & Derecho a una vivienda digna. & $\begin{array}{l}\text { Espacio, comodidad, servicios públi- } \\
\text { cos, durabilidad, seguridad, esparci- } \\
\text { miento y calidad de vida. }\end{array}$ \\
\hline $\begin{array}{l}\text { Política de gestión am- } \\
\text { biental urbana, 2008 } \\
\text { [21]. }\end{array}$ & $\begin{array}{l}\text { Establecer directrices para el manejo sos- } \\
\text { tenible de las áreas urbanas. }\end{array}$ & $\begin{array}{l}\text { Vivienda, espacio público, transpor- } \\
\text { te urbano, recurso hídrico, servicios } \\
\text { públicos y residuos. }\end{array}$ \\
\hline $\begin{array}{l}\text { Política de producción } \\
\text { y consumo sostenible, } \\
\text { 2010 [22]. }\end{array}$ & $\begin{array}{l}\text { Plantear estrategias para modificar los pa- } \\
\text { trones de producción y consumo. }\end{array}$ & Ciclo de vida. \\
\hline $\begin{array}{l}\text { Política pública de ur- } \\
\text { banismo y construcción } \\
\text { sostenible, 2014 [12]. }\end{array}$ & $\begin{array}{l}\text { Armonizar el urbanismo y las construc- } \\
\text { ciones. }\end{array}$ & $\begin{array}{l}\text { Suelo urbano, transporte y movili- } \\
\text { dad; servicios públicos, vías y espa- } \\
\text { cio público, VIS, edificaciones públi- } \\
\text { cas y edificaciones privadas. }\end{array}$ \\
\hline $\begin{array}{l}\text { Política pública de ur- } \\
\text { banismo y construcción } \\
\text { sostenibles del valle de } \\
\text { Aburrá, 2016 [18]. }\end{array}$ & $\begin{array}{l}\text { La política presenta dos niveles de indi- } \\
\text { cadores: } \\
\text { el primer nivel está constituido por indi- } \\
\text { cadores técnicos que permiten evaluar la } \\
\text { sostenibilidad en los diferentes ámbitos de } \\
\text { aplicación y las fases del ciclo de vida de } \\
\text { los proyectos constructivos. } \\
\text { El segundo nivel corresponde a los indi- } \\
\text { cadores de política pública los cuales per- } \\
\text { mitirán evaluar la efectividad de la imple- } \\
\text { mentación de la política pública. }\end{array}$ & $\begin{array}{l}\text { Se compone de cinco ejes: } \\
\text { Ecoeficiencia. } \\
\text { Habitabilidad. } \\
\text { Viabilidad. } \\
\text { Complejidad. } \\
\text { Resiliencia. } \\
\text { pos ejes de movilidad y equidad de- } \\
\text { nificación (POT), por lo cual no es- } \\
\text { tán incluidos en el modelo. }\end{array}$ \\
\hline
\end{tabular}


La primera de las políticas derivadas de la Constitución de 1991, y que tiene relación directa con los conceptos de vivienda, ciudad y medio ambiente, es la política de Ciudades amables, desarrollada por el Departamento Nacional de Planeación en el año 2006 y de la cual se valora la inclusión por primera vez del concepto de "ciudad sostenible" como aspiración del Estado colombiano, para lo cual plantea una serie de estrategias que dividen el entorno construido en cuatro componentes y cada uno cumple un papel en la aproximación al concepto de ciudad sostenible. Los componentes que dicha política incluye son: desarrollo urbano, vivienda, agua y saneamiento básico y transporte [23]. Cabe anotar que, a pesar de haber definido dichos componentes como estratégicos para alcanzar ciudades sostenibles, el no contar con instrumentos y mecanismos que condicionen el accionar de los diferentes sectores involucrados, no ha permitido que la política se materialice y genere cambios que permitan aproximarnos a ciudades sostenibles.

En el año 2008 se formula la Política de gestión ambiental urbana, la cual tenía como objetivo establecer directrices para el manejo sostenible de las áreas urbanas, buscando armonizar la gestión y las políticas sectoriales a través de espacios de coordinación interinstitucional y de participación ciudadana. "Dicha política estableció, como uno de sus objetivos de gestión, contribuir al mejoramiento de la calidad del hábitat urbano, con una meta específica enfocada a la definición y establecimiento de principios y lineamientos ambientales para el diseño y la construcción de vivienda sostenible" [10], todo ello enmarcado en una estrategia de calidad del hábitat urbano. La política antes mencionada presenta como debilidad el concentrarse únicamente en áreas urbanas, dejando de lado la sostenibilidad en el territorio rural y urbanorural. Dicha política propone, en contraposición con los ejes temáticos propuestos por la política de ciudades amables, cinco temas de importancia en la búsqueda de la sostenibilidad, estos son: vivienda, espacio público, transporte urbano, recurso hídrico, servicios públicos y residuos. Se observa cómo las temáticas que prioriza tienen relación directa con la infraestructura de soporte de los contextos urbanos, pero no pone en discusión otros aspectos de la sostenibilidad urbana relacionados con el modelo de ocupación, con el modelo de consumo, aprovechamiento de los recursos naturales o desarrollo humano. Igual que sucede en las políticas anteriores, adolece de falta de concreción y de formular programas precisos en las diferentes líneas temáticas.

Más recientemente, en el año 2010, se formuló la política de producción y consumo sostenible, política que aparentemente no tiene relación con la vivienda y la ciudad, sin embargo, lo más valorable de la misma es la comprensión de los problemas de insostenibilidad que están ligados a las actividades productivas, aborda el problema de la sostenibilidad desde una perspectiva de sectores productivos de oferta y demanda, 
reconoce la importancia de impactar los ciclos productivos y de consumo, tomando como referencia el ciclo de vida [23].

Esta política busca plantear estrategias para modificar los patrones de producción y consumo. En el caso particular del sector de la construcción la aplicación de la política implicará evaluar de dónde provienen las materias primas que se emplean, qué se hace con los residuos sólidos provenientes de la demolición, excavación y materiales empleados en la construcción de obras de infraestructura, buscando que la mayor parte de ellos se incorporen de nuevo al sistema productivo de la construcción en nuevos materiales y dentro de la misma obra en procesos constructivos diferentes al de origen.

La Política pública de urbanismo y construcción sostenible, la cual estuvo en proceso de perfeccionamiento y discusión desde el año 2013, presentaba un enfoque integral y multidimensional del problema. Sin embargo, en el año 2015 se expidió el Decreto 1285 de 2015, que reglamenta dicha política y tiene por objeto establecer lineamientos de construcción sostenible para edificaciones, encaminados al mejoramiento de la calidad de vida de los habitantes y al ejercicio de actuaciones con responsabilidad ambiental y social, objeto que se desarrolla a partir de generar medidas para el ahorro de agua y energía en las edificaciones. Este decreto deja de lado muchos de los aspectos que el documento preparatorio de la política había enunciado, y que podrían aportar a armonizar el urbanismo y las construcciones con la base biofísica y sociocultural leídos desde una perspectiva multidimensional [12].

Frente a la problemática habitacional, se ha planteado una solución de infraestructura que se ve reflejada en un concepto generalizado asociado a dicho problema -denominado política de vivienda-, en ella, la vivienda se entiende como la solución arquitectónica resultado de la aplicación de la norma. Allí radica el primer obstáculo en la visión del problema, ya que concebirlo únicamente como un asunto de vivienda, implica sesgar tanto la lectura como la construcción de estrategias de solución solo a lo que atañe a la dimensión fisicoespacial, lo que lleva a concentrar de manera particular el interés en entregar una vivienda como respuesta al déficit.

"De igual forma, somos conscientes de que la mirada debe ser ampliada en un mayor sentido de integralidad, pues no se puede desconocer que el entorno creado de la ciudad incide directamente en el desarrollo de la vivienda y que la ocupación y consolidación de los sectores residenciales influye a su vez en el desarrollo y expansión de la ciudad. Así, la calidad de la vivienda está asociada al entorno urbano y a la satisfacción de las necesidades inherentes de movilidad, recreación, abastecimiento, trabajo, educación, salud y demás factores que requieren el desarrollo social y ambiental de los ciudadanos y las áreas urbanas" [10]. 
En Colombia existen pocos ejemplos de propuestas de vivienda sostenible, la mayoría de ellas se han desarrollado en la zona rural, por ejemplo, iniciativas privadas (tal es el caso de los concursos desarrollados por la Fundación Corona y la Revista Escala que se enfocan en propuestas que buscan generar un hábitat sostenible en contextos urbanos y rurales) y que en algunos casos hacen énfasis en la recuperación de materiales y sistemas constructivos tradicionales que ofrecen adecuadas condiciones para garantizar confort térmico y lumínico haciendo uso de materiales locales. También en el último año, se ha visto la afluencia de proyectos de vivienda de estratos altos que buscan la certificación LEED, como una forma de presentar un valor agregado en un mercado inmobiliario muy competitivo, donde la construcción sostenible se vuelve una aspiración que da estatus a la vivienda y la posiciona con relación a sus iguales.

\section{RESULTADOS}

El desarrollo sostenible se entiende como una aspiración que, llevada al campo de la construcción sostenible, se ve aplicada a diferentes escalas. Para el caso de la vivienda implicará una lectura de la sostenibilidad que interrelacione diferentes escalas involucradas así: la escala territorial al concepto de ciudades sostenibles, la escala urbana, al de sostenibilidad urbana y la escala de intervención micro al concepto de arquitectura sostenible. Adicionalmente, hablar de sostenibilidad de la vivienda implica vincular también los estructurantes naturales de la realidad con los estructurantes artificiales, de tal manera que cualquier propuesta que evalúe la sostenibilidad de la vivienda implica una perspectiva sistémica donde los elementos a considerar hacen parte de las diferentes interrelaciones.

La manera como se han desarrollado los asentamientos humanos, y la ciudad como su mayor expresión, han llevado a que el no considerar los determinantes ambientales y sociales en la producción del hábitat, ocasione múltiples desequilibrios y problemáticas socioambientales.

Si se quiere aspirar a permear la arquitectura y los asentamientos humanos con el concepto de sostenibilidad, es necesario cambiar el paradigma que tradicionalmente define la vivienda como técnica arquitectónica y derivar hacia un nuevo paradigma que entienda la vivienda en relación con su entorno, en el que la producción de hábitat no se circunscriba a la solución arquitectónica y donde, partiendo del concepto internacional de desarrollo sostenible, se entienda que pensar en lo sostenible es un asunto que supera los límites de lo arquitectónico e interactúa y modifica los sistemas colindantes al mismo: entorno urbano, ciudad y territorio.

Desde la perspectiva normativa, que reglamenta el actuar en el tema de vivienda en el país, se rescata lo propuesto en las diferentes políticas públicas analizadas así: 
- Constitución política de Colombia (1991): calidad en el diseño de la vivienda y el urbanismo, búsqueda de una vivienda digna (segura, sana, confortable) [20].

- Ciudades amables: se asegura la sostenibilidad ambiental en el entorno construido a través de cuatro componentes: desarrollo urbano, vivienda, agua y saneamiento básico y trasporte.

- Gestión ambiental urbana: se busca con ella garantizar la calidad del hábitat urbano a partir de analizar los siguientes factores: vivienda, espacio público, transporte urbano, recurso hídrico, servicios públicos y residuos [21].

- Urbanismo y construcción sostenible: se desarrolla en dos componentes, el primero urbanismo, que evalúa factores como: suelo urbano transporte y movilidad. El segundo construcciones, considera factores: VIS, edificaciones públicas y edificaciones privadas [12].

Se observa que todas las políticas consideran unos temas en común, lo que le otorga relevancia a los mismos e implica considerarlos en el modelo a proponer, estos son: vivienda, saneamiento básico y transporte. Se requiere además superar el nivel de la política de tal manera que se generen instrumentos que hagan viable la inspiración planteada por la política y no quedarnos en enunciados teóricos que no transforman, ni impactan el desarrollo humano de la población.

\section{CONCLUSIONES}

Los conceptos asociados a la arquitectura sostenible, y reconocidos a nivel internacional, buscan una inserción armónica del elemento construido en el contexto natural, un uso eficiente de los recursos naturales requeridos para su funcionamiento empleando, en algunos casos, el análisis del ciclo de vida de los materiales como estrategia para evaluar los mismos desde su origen hasta su conclusión como residuo sólido. En el caso particular de la arquitectura bioclimática, esta se concentra en apostar al confort térmico y bienestar como indicadores de coherencia con el contexto. Aunque la mayor parte de dichas propuestas conceptuales abordan el contexto biofísico como determinante, ninguna incluye el contexto sociocultural como condicionante de la respuesta urbana y arquitectónica, por esta razón las soluciones que se entregan son extrapolables a contextos biofísicos similares, lo que hace que pierdan su carácter específico y particular.

No ha habido una conciencia de ocupación del territorio en coherencia con las restricciones ambientales del mismo, se entiende el suelo como aquellas áreas no 
ocupadas por infraestructura y que son susceptibles de admitir un módulo de habitación, sea este informal, de invasión o formal. Es por esto que los recursos naturales se ven afectados, ya que elementos como los retiros de quebradas no se consideran como áreas de protección necesarias para evitar riesgo por inundación, sino que son tomadas como áreas disponibles para la población que llega desplazada y no encuentra otro lugar que le sea asequible.

\section{REFERENCIAS}

[1] Organización de las Naciones Unidas, "Informe de la Conferencia de las Naciones Unidas sobre el medio ambiente y el desarrollo," Río de Janeiro, 3 a 14 de junio de 1992, vol. I, 1992.

[2] Organización de las Naciones Unidas, Informe Brundtland "Nuestro futuro común”, 1987.

[3] Unión Internacional de Arquitectos, Informe Definición de la UIA International Union of Architects y la AIA American Institute of Architects, 1993.

[4] United States Agency for International Development -Usaid, Documento técnico de soporterecomendaciones de política pública de construcción y urbanismo sostenible informe final, AECOM, Bogotá, 2013.

[5] Colombia. Ministerio de Ambiente, Vivienda y Desarrollo Territorial, Anexo 1. "Guía de construcción sostenible para el ahorro de agua y energía en edificaciones, Bogotá, Colombia," [En línea], vol. 85, Disponible: http://www.minvivienda.gov.co/Documents/ViceministerioVivienda/ANEXO\%201\%200549\%20-\%202015.pdf, 2016.

[6] Ministerio de Vivienda y Urbanismo, Satisfacción residencial en la vivienda básica, SERVIU: "La perspectiva de capital social," Santiago de Chile, [En línea], pp. 54, Disponible: http://www.minvu.cl/incjs/download.aspx?glb_cod_nodo=20070411164455\&hdd_nom archivo=Satisfacci\%C3\%B3n\%20Residencial\%20Capital\%20Social.pdf, 2001.

[7] M. M. Garcilaso de la Vega, "Desarrollo sistémico de la política de vivienda en Cuba", Arquitectura y Urbanismo, vol. XXXII, N. ${ }^{\circ}$ 2, pp. 16-20, 2011.

[8] C. Gómez Gutiérrez, y A. Gómez Sal, Referencias para un análisis del desarrollo sostenible, ESPA: Universidad de Alcalá, 2013, p. 200.

[9] Banco Interamericano de Desarrollo-BID, "Metodología ciudades emergentes y sostenibles," 3. ${ }^{a}$ ed., [En línea], p. 172, Disponible: https://publications.iadb.org/bitstream/handle/11319/8119/ Guia-Metodologica-Programa-de-Ciudades-Emergentes-y-Sostenibles-Tercera-edicion.pdf, 2016.

[10] Colombia, Ministerio de Ambiente y Desarrollo Sostenible, "Criterios ambientales para el diseño y construcción de vivienda urbana,” Bogotá, [En línea], p. 200, Disponible: http://www. minambiente.gov.co/images/AsuntosambientalesySectorialyUrbana/pdf/Sello_ambiental_colombiano/cartilla_criterios_amb_diseno_construc.pdf, 2012.

[11] R. Quiroga Martínez, "Indicadores regionales de desarrollo sostenible: avances y perspectivas para América Latina y el Caribe,” CEPAL, Santiago de Chile, [En línea], p. 228, Disponible: 
http://repositorio.cepal.org/bitstream/handle/11362/5498/S0700589_es.pdf;jsessionid=D3138 9A3C391422D6C66BCCF7802088C?sequence=1, 2007.

[12] Alcaldía Mayor de Bogotá D.C., "Documento técnico de soporte política pública de ecourbanismo y construcción sostenible de Bogotá," [En línea], p. 51, Disponible: http://www.sdp.gov.co/ portal/page/portal/PortalSDP/Normativa/ProyectosDecreto/Politica_Publica_Ecourbanismo/ DTS_PPECS_V5_140526_1.pdf, 2014.

[13] E. Higueras, “El reto de la ciudad habitable y sostenible,” [En línea], Dapp, p. 226, Disponible: http://www.coag.es/websantiago/pdf/ester_higueras.pdf

[14]. BCN Ecología \&AL21, "Sistema de indicadores y condicionantes para ciudades grandes y Medianas," [En línea], p. 81, Disponible: http://www.magrama.gob.es/es/calidad-y-evaluacionambiental/temas/medio-ambiente-urbano/INDICADORES_CIUDADES_GRANDES_Y_MEDIANAS_tcm7-177731.pdf, 2013.

[15] Colombia, Ministro del Interior y Ministro de Justicia, Decreto 2190 de 2009, Bogotá, 2009

[16] Colombia, Ministro de Defensa Nacional, Decreto 1285 de 2015, Bogotá, 2015.

[17] J. E. Reyes Nieto, L. Simões da Silva, V. Murtinho, C. Rigueiro, H. Gervasio, Y A. Bettencourt, "Desarrollo de una metodología de evaluación integral de sustentabilidad a nivel urbano", Portugal, [En línea], pp. 1669-1678, Disponible: https://www.researchgate.net/ profile/Jocelyn_Reyes_Nieto/publication/307977714_Desarrollo_de_una_metodologia_de_ evaluacion_integral_de_sustentabilidad_a_nivel_urbano/links/57d56ebf08ae601b39aa6c44. pdf?origin=publication_list

[18] Área Metropolitana del Valle de Aburrá, Política pública de construcción sostenible, Medellín, 2016.

[19] Colombia. Ministerio de Ambiente, Vivienda y Desarrollo Territorial, "Calidad en la vivienda de interés social. Serie Guías de asistencia técnica para vivienda de interés social," Bogotá, Colombia, [En línea], p. 61, Disponible: http:/www.minvivienda.gov.co/Documents/ guia_asis_tec_vis_1.pdf, 2011.

[20] Constitución Política de Colombia, Bogotá, 1991.

[21] Colombia, Ministerio de Ambiente y Vivienda y Desarrollo Sostenible, "Política de Gestión Ambiental Urbana,” Bogotá, [En línea], p. 54, Disponible: http:/www.minambiente.gov.co/images/AsuntosambientalesySectorialyUrbana/pdf/Polit\%C3\%ACcas_de_la_Direcci\%C3\%B3n/ Politica_de_Gestion_Ambiental_Urbana.pdf, 2008.

[22] Colombia, Ministerio de Ambiente y Vivienda y Desarrollo Sostenible, "Política de producción y consumo sostenible," Bogotá, [En línea], p. 72, Disponible: http://www. minambiente.gov.co/images/AsuntosambientalesySectorialyUrbana/pdf/Polit $\% \overline{\mathrm{C} 3 \% \mathrm{ACcas}}$ de_la_Direcci\%C3\%B3n/Politica_de_Gestion_Ambiental_Urbana.pdf, 2010.

[23] Presidencia de la República de Colombia, Construir ciudades amables, propuesta para discusión, Bogotá: Departamento Nacional de Planeación, y Ministerio de Ambiente y Vivienda y Desarrollo Sostenible, p. 108, 2006. 